\title{
Pseudorapidity profile of transverse momentum fluctuations in heavy ion collisions
}

\author{
Sandeep Chatterjef ${ }^{*}$ and Piotr Bożek $\dagger^{\dagger}$ \\ AGH University of Science and Technology, \\ Faculty of Physics and Applied Computer Science, \\ al. Mickiewicza 30, 30-059 Krakow, Poland
}

\begin{abstract}
We investigate pseudorapidity correlations of the average transverse flow of particles emitted in relativistic heavy-ion collisions. We employ $3+1$ dimensional viscous relativistic hydrodynamics with initial conditions from the quark Glauber Monte Carlo model to confront the recent measurements on the pseudorapidity correlations of the transverse momentum fluctuations in $\mathrm{Pb}+\mathrm{Pb}$ collisions at $\sqrt{s_{N N}}=2760 \mathrm{GeV}$. We find good agreement between the model predictions and data. Further, we study two other observables build with the covariance of the average transverse momentum in different rapidity bins. These observables have better stability under various systematics, thus allowing for a robust comparison between data and model. The transverse flow-transverse flow correlation coefficient is directly related to correlations of the underlying collective flow at different pseudorapidities. The 3-bin measure of $p_{T}$ factorization breaking in pseudorapidity gives an estimate of possible decorrelation of the average transverse flow in the longitudinal direction.
\end{abstract}

\section{INTRODUCTION}

Relativistic heavy ion collisions produce a hot and dense strongly interacting fireball that rapidly expands and freezes into free-streaming hadrons that are registered by the detectors. There has already been enormous progress in understanding the transverse (to the beam direction) evolution of the initial transverse spatial anisotropy of the overlap zone into the final state momentum anisotropy through equations of viscous relativistic hydrodynamics [1. An analogous effort to comprehend the longitudinal dynamics has picked up pace as well. It is widely believed on the basis of causality arguments that correlations over large rapidity gaps arise due to early time dynamics. Thus a good knowledge of the rapidity profile of the fireball will lead to a better understanding of the initial conditions and early dynamics in the longitudinal direction.

There have been several studies on pseudorapidity correlations of event-plane orientations both in theory [2 6] as well as in experiments [7, 8. Further, multiplicity correlation at different rapidities have been studied as well [9. However, the latter is more challenging as it is difficult to disentangle different sources of short and long range correlations.

Fluctuations and correlation of transverse momentum are believed to give insight into the mechanism of energy deposition in heavy ion collisions and could serve as a probe of the properties of the medium formed [10 27. Collective expansion of the fireball can be at the origin of fluctuations of the average transverse momentum of particles emitted in an event 28 30. In events where the initial transverse size of the fireball is larger, a smaller transverse flow is generated. Experimentally, fluctuations of the average transverse momentum have be analyzed in heavy-ion and proton-proton collisions [31 35].

\footnotetext{
* Sandeep.Chatterjee@fis.agh.edu.pl

$\dagger$ piotr.bozek@fis.edu.agh.pl
}

Hydrodynamic models with Monte Carlo Glauber initial conditions predict sizable transverse momentum fluctuations, in semiquantitative agreement with the data.

In this paper we study correlations of the average transverse momentum in different pseudorapidity bins. The decorrelation of the average transverse momentum in two pseudorapidity bins is a measure of the factorization breaking of the flow. In the hydrodynamic model such a factorization breaking is possible due to event-by-event fluctuations. The ALICE collaboration has reported preliminary measurement of pseudorapidity correlations of average transverse momenta at different pseudorapidities 36. The correlation of the average transverse momenta has been also calculated in some event generators 37 . We calculate this observable in the hydrodynamic framework and find a fair agreement with data.

In order to reduce strong statistical fluctuations we propose to measure a modified correlation coefficient between transverse momenta in different pseudorapidity bins, removing the self-correlation contribution to the variance [31. This modified definition could be directly interpreted as a measure of transverse flow-transverse flow In order to reduce non-flow contributions a 3-bin measure of the transverse momentum decorrelation can be used, in analogy to the one used for the azimuthal flow coeffcients [7. In the hydrodynamic model with Monte Carlo Glauber initial conditions, the decorrelation of the collective average transverse momentum is $0.8-1.0$ for pseudorapidities $|\eta|<2.5$. Additional non-flow effects in experimental estimates can modify these values.

\section{GLAUBER MODEL AND VISCOUS HYDRODYNAMICS}

We use event-by-event viscous relativistic hydrodynamics with fluctuating initial conditions $[38$. The initial conditions are take from a Monte Carlo Glauber model. We use the quark Glauber model, where each nucleon is composed of three quarks ( details and parameters can be 
found in [39). The Monte Carlo Glauber model predicts for each event the positions of the participant quarks. The initial entropy density is deposited at the participant positions in the form of Gaussians $g_{i}(x, y)$ of width $0.3 \mathrm{fm}$ in the transverse plane.

The longitudinal profile used in our study involves asymmetric deposition in space-time rapidity $\eta_{\|}$from left or right going participants. Event-by-event fluctuations in the number of left and right going participants $\left(N_{-}\right.$ and $N_{+}$respectively) or their positions leads to a decorrelation of the flow and entropy at different rapidities

$$
s\left(x, y, \eta_{\|}\right)=\sum_{i=1}^{N_{+}} g_{i}(x, y) f_{+}\left(\eta_{+}\right)+\sum_{i=1}^{N_{-}} g_{i}(x, y) f_{-}\left(\eta_{\|}\right) .
$$

The longitudinal density profile is

$$
f_{ \pm}\left(\eta_{\|}\right)=\frac{y_{\text {beam }} \pm \eta_{\|}}{2 y_{\text {beam }}} H\left(\eta_{\|}\right) \text {for }\left|\eta_{\|}\right|<y_{\text {beam }},
$$

and 40.

$$
H\left(\eta_{\|}\right)=\exp \left(-\frac{\left(\left|\eta_{\|}\right|-\eta_{p}\right)^{2} \Theta\left(\left|\eta_{\|}\right|-\eta_{p}\right)}{2 \sigma_{\eta}^{2}}\right),
$$

where $\sigma_{\eta}=1.4$ and $\eta_{p}=1.15$. Model calculations presented below illustrate the application of the transverse momentum correlation and do not represent an extensive study of possible scenarios for fluctuations in the initial state or in the dynamics. We note that in other models of the initial state the initial decorrelation in space-time rapidity may be larger [3, 4, 41, 42, The initial fireball expands collectively. We set the shear viscosity to entropy ratio to 0.08 . At the freeze-out temperature of $150 \mathrm{MeV}$ statistical emission of particles occurs 43] (particlization procedure). This framework predicts correctly the fluctuations of the average transverse momentum in $\mathrm{Pb}+\mathrm{Pb}$ collisions [44, which is a good starting point to investigate $p_{T}$ correlations in pseudorapidity.

Generated events have realistic multiplicities and involve non-flow correlations from resonance decays. Alternatively, we generate events with oversampled multiplicity for each freeze-out hyperspace. In such events non-flow effects are reduced and one measures directly fluctuations and correlations of the average transverse flow in the spectra.

\section{PEARSON CORRELATION COEFFICIENT}

In this study, we focus on the statistical properties of the average transverse momentum in the event $\left[p_{T}\right]$

$$
\left[p_{T}\right]=\frac{1}{n} \sum_{i} p_{i}
$$

where $n$ is the number of particles in the event in a given acceptance window ( $p_{i}$ is the transverse momentum of particle $i$ ). Thus, [...] denotes averaging over the particles in an event. Similarly, the event average $\langle\ldots\rangle$ of $\left[p_{T}\right]$ could be defined as

$$
\left\langle\left[p_{T}\right]\right\rangle=\frac{1}{N_{e v}} \sum_{e v}\left[p_{T}\right]
$$

where $N_{e v}$ is the total number of events in the ensemble. We look at the correlation of $\left[p_{T}\right]$ in different pseudorapidity bins. The average transverse momentum is calculated for $p_{T}$ in the range $0.2-2.0 \mathrm{GeV}$. The observable $b\left(\left[p_{T}\right]_{F},\left[p_{T}\right]_{B}\right)$ that was measured by the ALICE collaboration is defined as 36

$$
\begin{aligned}
b\left(\left[p_{T}\right]_{F},\left[p_{T}\right]_{B}\right) & =\frac{\operatorname{Cov}\left(\left[p_{T}\right]_{F},\left[p_{T}\right]_{B}\right)}{\sqrt{\operatorname{Var}\left(\left[p_{T}\right]_{F}\right)} \sqrt{\operatorname{Var}\left(\left[p_{T}\right]_{B}\right)}} \\
& =\frac{\left\langle\left(\left[p_{T}\right]_{F}-\left\langle\left[p_{T}\right]\right\rangle_{F}\right)\left(\left[p_{T}\right]_{B}-\left\langle\left[p_{T}\right]\right\rangle_{B}\right)\right\rangle}{\sqrt{\left\langle\left(\left[p_{T}\right]_{F}-\left\langle\left[p_{T}\right]\right\rangle_{F}\right)^{2}\right\rangle} \sqrt{\left\langle\left(\left[p_{T}\right]_{B}-\left\langle\left[p_{T}\right]\right\rangle_{B}\right)^{2}\right\rangle}} \\
& =\frac{\left\langle\frac{1}{n_{F}} \sum_{i \in F}\left(p_{i}-\left\langle\left[p_{T}\right]\right\rangle_{F}\right) \frac{1}{n_{B}} \sum_{j \in B}\left(p_{j}-\left\langle\left[p_{T}\right]\right\rangle_{B}\right)\right\rangle}{\sqrt{\left\langle\frac{1}{n_{F}^{2}} \sum_{i, j \in F}\left(p_{i}-\left\langle\left[p_{T}\right]\right\rangle_{F}\right)\left(p_{j}-\left\langle\left[p_{T}\right]\right\rangle_{F}\right)\right\rangle} \sqrt{\left\langle\frac{1}{n_{B}^{2}} \sum_{i, j \in B}\left(p_{i}-\left\langle\left[p_{T}\right]\right\rangle_{B}\right)\left(p_{j}-\left\langle\left[p_{T}\right]\right\rangle_{B}\right)\right\rangle}}
\end{aligned}
$$

where $F \in\left[\eta_{\min }, \eta_{\max }\right]$ and $B \in\left[-\eta_{\max },-\eta_{\min }\right]$ denote the two pseudorapidity bins in the forward(F) and backward(B) hemispheres whose correlation strength is being studied. Mathematically, the quantity $b\left(\left[p_{T}\right]_{F},\left[p_{T}\right]_{B}\right)$ represents the Pearson correlation coefficient between $\left[p_{T}\right]_{F}$ and $\left[p_{T}\right]_{B}$ averaged over the ensemble of events.

In Fig. 1 are shown preliminary results for the Pearson correlation coefficient $b\left(\left[p_{T}\right]_{F},\left[p_{T}\right]_{B}\right)$ measured in $\mathrm{Pb}+\mathrm{Pb}$ collisions at different centralities. The centrality dependence of the correlation coefficient is nonmonotonous, the correlation is maximal for semi-central collisions. Interestingly the calculation in the hydrodynamic model with quark Glauber initial conditions (filled symbols in Fig. 1) reproduces fairly well the magnitude of the correlation and quantitatively it shows a similar centrality dependence. The correlation coefficient 


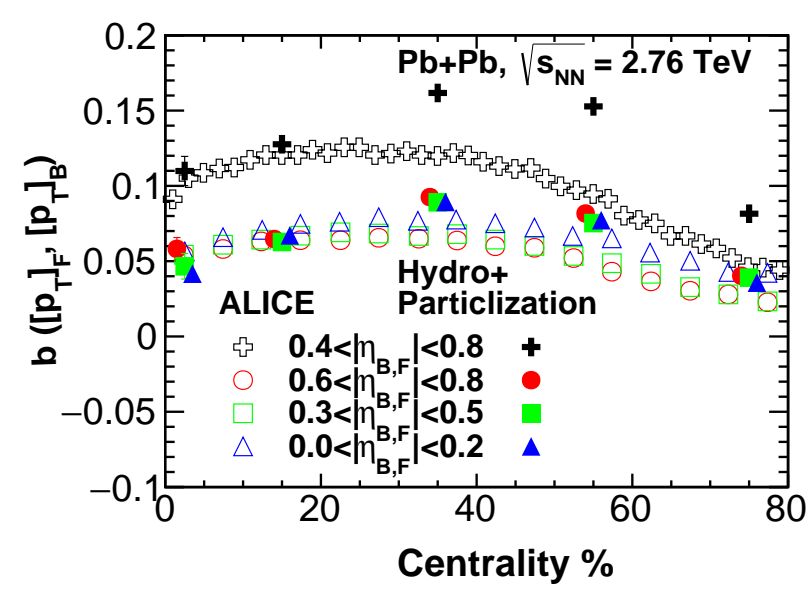

FIG. 1. (Color online) Preliminary data from ALICE Collaboration [36] on Pearson correlation coefficient $b\left(\left[p_{T}\right]_{F},\left[p_{T}\right]_{B}\right)$ in $\mathrm{Pb}+\mathrm{Pb}$ collisions at $\sqrt{s}=2760 \mathrm{GeV}$ are compared to hydrodynamic model predictions. Results are shown for different choices of pseudorapidity bins where the average transverse momentum is calculated.

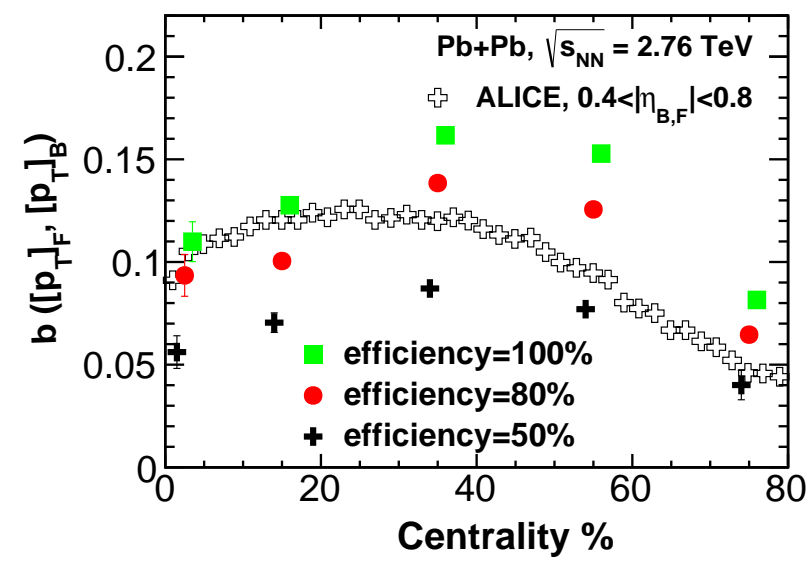

FIG. 2. (Color online) Preliminary data from ALICE Collaboration [36] on the correlation coefficient $b\left(\left[p_{T}\right]_{F},\left[p_{T}\right]_{B}\right)$ in two pseudorapidity bins $\left(0.4<\left|\eta_{F, B}\right|<0.8\right)$ in $\mathrm{Pb}+\mathrm{Pb}$ collisions at $\sqrt{s}=2760 \mathrm{GeV}$. Hydrodynamic model results are shown for $100 \%$ (squares), $80 \%$ (circles) and $50 \%$ efficiency (crosses).

$b\left(\left[p_{T}\right]_{F},\left[p_{T}\right]_{B}\right)$ shows a strong dependence on the width of the pseudorapidity bin used. On the other hand, the dependence on the separation of the two bins is weak. It is difficult to interpret the experimental data for this quantity as the result depends strongly on the chosen bin width.

As shown in Fig. 2 the results depend also on the efficiency. A change from of the efficiency from $100 \%$ to $80 \%$ or $50 \%$ in the model calculation shifts the value of $b\left(\left[p_{T}\right]_{F},\left[p_{T}\right]_{B}\right)$ significantly. The reason for this behavior is a strong dependence of the variance $\operatorname{Var}\left(\left[p_{T}\right]\right)$ in Eq. (6) on multiplicity. As argued in Ref. 31] this quantity contains a contribution from statistical fluctuations in the sampling of transverse momenta from a spectrum. It

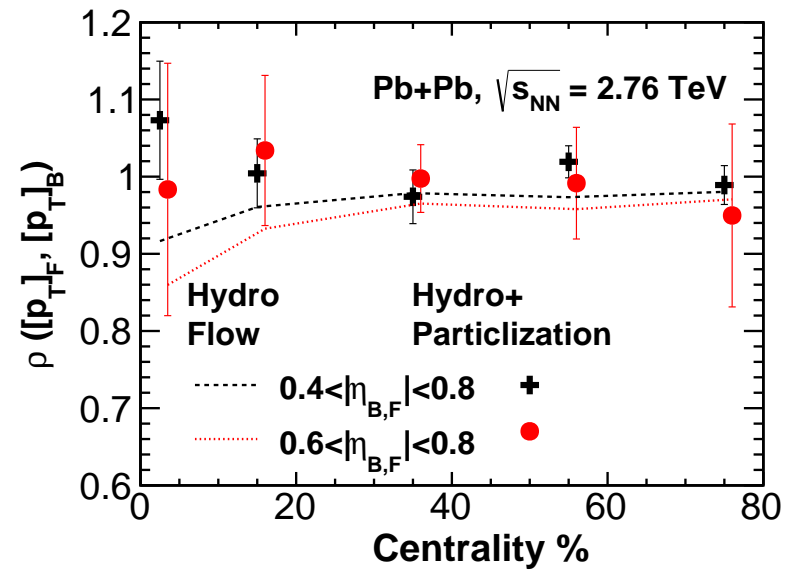

FIG. 3. (Color online) Transverse flow-transverse flow correlation coefficient $\rho\left(\left[p_{T}\right]_{F},\left[p_{T}\right]_{B}\right)$ predicted in the hydrodynamic model plotted as a function of collision centrality. Results are shown for two choices of pseudorapidity intervals $0.4<\left|\eta_{B, F}\right|<0.8$ (crosses and dashed line) and $0.6<\left|\eta_{B, F}\right|<0.8$ (circles and dotted line). Symbols represent the results obtained from events generated with realistic multiplicities and non-flow correlations from resonance decays, while the lines are obtained by integrating the particle spectra and include only correlations from flow.

cannot be a measure of the fluctuations of the transverse flow. This effect is especially important when using small bin widths.

\section{TRANSVERSE FLOW-TRANSVERSE FLOW CORRELATION COEFFICIENT}

The variance of the average transverse momentum of a spectrum can be estimated when using a formula excluding self-correlations

$$
C_{p_{T}}=\frac{1}{n(n-1)} \sum_{i \neq j}\left(p_{T}^{i}-\left[p_{T}\right]\right)\left(p_{T}^{j}-\left[p_{T}\right]\right)
$$

For independent particle emission, this formula eliminates statistical fluctuations from the estimate of the variance of the transverse flow. It has been used in experimental measurements of the $p_{T}$ fluctuations [31 35] and to define the transverse momentum-flow correlation coefficient 45 .

The correlation coefficient for the average transverse flow in two pseudorapidity bins takes the form

$$
\rho\left(\left[p_{T}\right]_{F},\left[p_{T}\right]_{B}\right)=\frac{\operatorname{Cov}\left(\left[p_{T}\right]_{F},\left[p_{T}\right]_{B}\right)}{\sqrt{C_{p_{T}}^{F} C_{p_{T}}^{B}}}
$$

where self-correlations are excluded in the calculation of the variances in the denominator. If only flow correlations are present, the correlation coefficient (8) is smaller than 1. As discussed for the factorization breaking of azimuthal flow coefficients, a value larger than 1 signals a significant contribution from non-flow correlations [46]. 


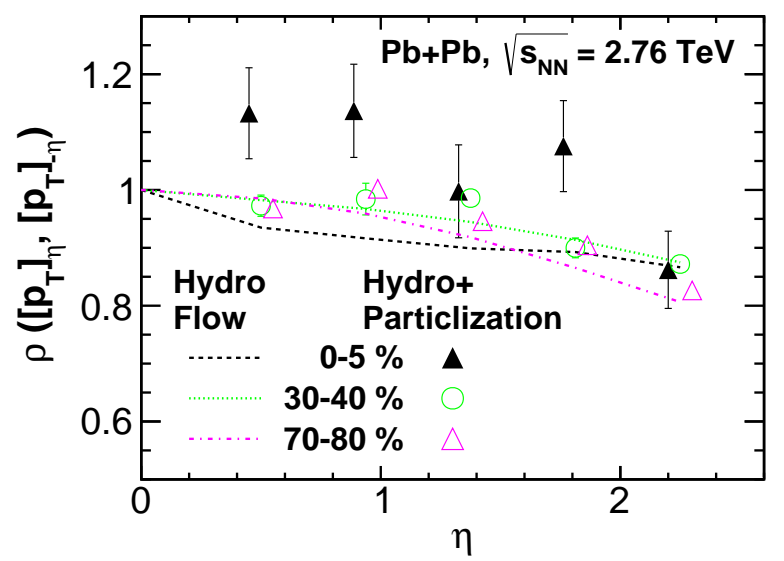

FIG. 4. (Color online) Transverse flow-transverse flow correlation coefficient $\rho\left(\left[p_{T}\right]_{\eta},\left[p_{T}\right]_{-\eta}\right)$ plotted plotted as a function of $\eta$ for three different centralities. Symbols are for results from realistic finite multiplicity events and lines are obtained from spectra integration (see caption of Fig. 3).

Fig. 3 presents the results for the transverse flowtransverse flow correlation coefficient as a function of centrality for the same kinematic range as the observable $b\left(\left[p_{T}\right]_{F},\left[p_{T}\right]_{B}\right)$ discussed in the previous section. The striking result is that $\rho\left(\left(\left[p_{T}\right]_{F},\left[p_{T}\right]_{B}\right)\right.$ is very different from $b\left(\left[p_{T}\right]_{F},\left[p_{T}\right]_{B}\right)$. The correlation coefficient $\rho$ is close to $1,8-10$ times larger than $b\left(\left[p_{T}\right]_{F},\left[p_{T}\right]_{B}\right)$. The only difference between the two observables is in the definition of the variance of the transverse momentum. In the limit of infinite multiplicity both quantities should be equal. In the hydrodynamic model, genuine correlations of the collective transverse flow can be calculated using oversampled events. The results are shown with lines in Fig. 3. The correlation coefficient for the collective transverse flow is always smaller than 1 . The factorization breaking of the average transverse flow in our hydrodynamic calculation is small; $\rho\left(\left(\left[p_{T}\right]_{F},\left[p_{T}\right]_{B}\right)>0.8\right.$. We notice that the decorrelation is stronger when the separation between the two pseudorapidity bins increases. In realistic events non-flow correlations from resonance decays are present. These non-flow correlations increase the value of $\rho\left(\left(\left[p_{T}\right]_{F},\left[p_{T}\right]_{B}\right)\right.$ with respect to the values corresponding to collective flow only. However, no definite conclusions on deviations of the correlation coefficient from 1 can be made with the statistics available in our simulations.

The correlation coefficient can be calculated for larger pseudorapidity intervals. We move the two pseudorapidity bins in the range $|\eta|<2.5$. The results for the correlation coefficient $\rho\left(\left(\left[p_{T}\right]_{\eta},\left[p_{T}\right]_{-\eta}\right)\right.$ as a function of the bin position $\eta$ are shown in Fig. 4 for three different centralities. For centralities $30-40 \%$ and $70-80 \%$ the decorrelation of the average transverse momenta deviates from 1 for bin separation $2 \eta>3$. It indicates that the decorrelation of the transverse flow could be observed in experiments using bins with a large pseudorapidity separation. With sufficient statistics, non-flow ef-

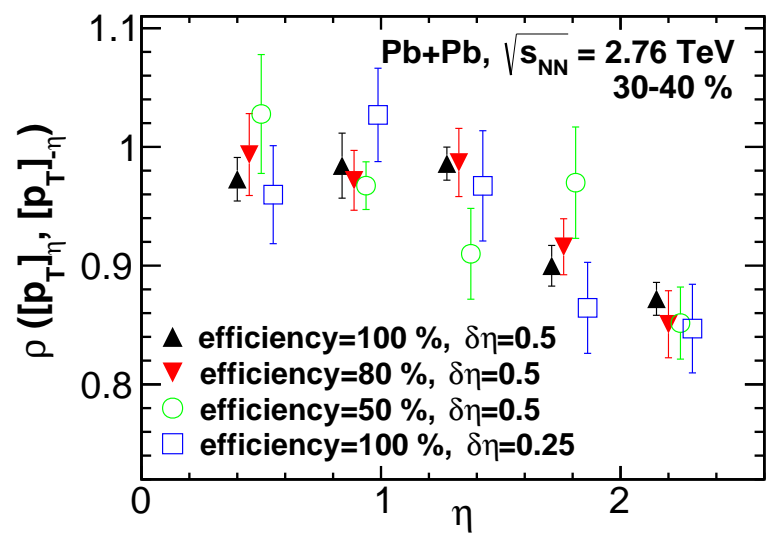

FIG. 5. (Color online) Transverse flow-transverse flow correlation coefficient $\rho\left(\left[p_{T}\right]_{\eta},\left[p_{T}\right]_{-\eta}\right)$ plotted as a function of $\eta$. We compare results of hydrodynamic calculations with assumed efficiency $100 \%, 80 \%$, and $50 \%$, all with bin width $\delta \eta=0.5$. Squares denote the results obtained with bin of width 0.25 and efficiency $100 \%$.

fects could be estimated by comparing results using sameand opposite-charged pairs.

We illustrate the robustness of the definition of the transverse flow-transverse flow correlation coefficient (Eq. 8) by comparing results obtained for different effciencies and bin widths. As shown in Fig. 5 the correlation coefficients obtained using $100 \%, 80 \%$, and $50 \%$ effciencies are compatible within statistical errors. Further, the results from $\eta$ bin widths of 0.5 and 0.25 also agree with each other. Thus, the fluctuations of transverse flow calculated without self-correlations (Eq. 7) have the desired property that the measured values do not depend on the multiplicity of the subsample used for the calculation.

The average transverse momentum in a pseudorapidity bin is an intensive quantity. It suggests to define the scaled covariance of the average transverse momenta as

$$
\frac{\operatorname{Cov}\left(\left[p_{T}\right]_{\eta},\left[p_{T}\right]_{-\eta}\right)}{\left\langle\left[p_{T}\right]\right\rangle_{\eta}\left\langle\left[p_{T}\right]\right\rangle_{-\eta}}
$$

The above quantity shows a very strong centrality dependence, similar to the scaled transverse momentum fluctuations [35, 44]

$$
\frac{\sqrt{C_{p_{T}}}}{\left\langle\left[p_{T}\right]\right\rangle} \simeq\left(\frac{d N}{d \eta}\right)^{-1 / 2}
$$

The above scaling is expected if transverse momentum fluctuations come from a superposition of independent sources. We note that experimental data and hydrodynamic model results show systematic deviations from this trend. In Fig. 6 is shown the scaled variance

$$
\frac{\operatorname{Cov}\left(\left[p_{T}\right]_{\eta},\left[p_{T}\right]_{-\eta}\right)}{\left\langle\left[p_{T}\right]\right\rangle_{\eta}\left\langle\left[p_{T}\right]\right\rangle_{-\eta}} \sqrt{\frac{d N^{\eta}}{d \eta} \frac{d N^{-\eta}}{d \eta}}
$$




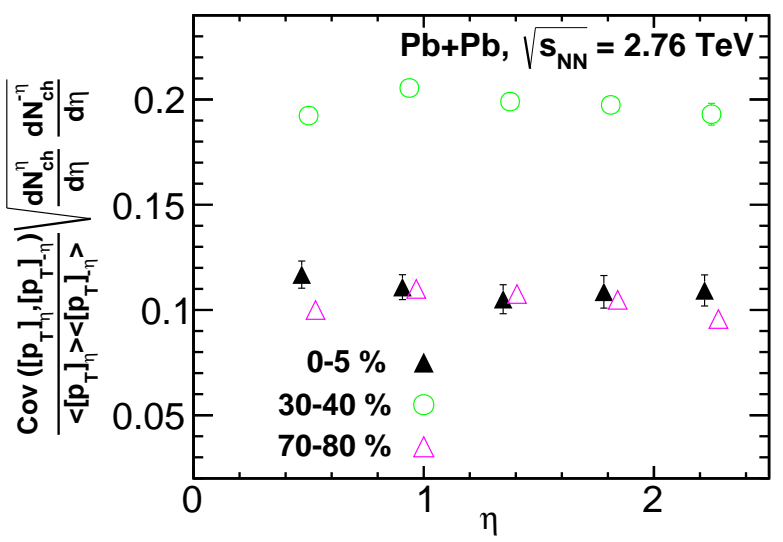

FIG. 6. (Color online) Covariance $\operatorname{Cov}\left(\left[p_{T}\right]_{\eta},\left[p_{T}\right]_{-\eta}\right)$ of the average transverse momentum in two pseudorapidity bins scaled by the product of the square roots of the charged particle densities and by the inverse of the average transverse momenta in the two pseudorapidity bins. Results are shown as a function of the bin position $\eta$, for three different centralities.

for three different centralities. One notices a weak dependence of this quantity on the separation of pseudorapidity bins. The remaining dependence on centrality of the magnitude of the scaled covariance can be explained by the deviation of the transverse momentum fluctuations from the scaling Eq. 10 . Unlike the correlation coefficient $\rho\left(\left[p_{T}\right]_{\eta},\left[p_{T}\right]_{-\eta}\right)$, the scaled covariance (11) can be calculated without involving the variance of the transverse flow. However, there is no simple physical interpretation of this quantity in the hydrodynamic model. Moreover, it is difficult to disentangle the dependence on the bin separation in pseudorapidity of the covariance from those of the average transverse momentum and charged particle density.

\section{3-BIN DECORRELATION MEASURE}

The transverse flow-transverse flow correlation coefficient (8) is plagued with non-flow contributions. The non-flow contribution to the covariance is reduced for widely separated pseudorapidity bins. On the other hand, the $p_{T}$ fluctuations measure $C_{p_{T}}$ has always a contribution from non-flow correlations. The CMS Collaboration has proposed to use a 3-bin decorrelation measure for azimuthal flow coefficients [7]. A similar decorrelation for transverse flow correlations in pseudorapidity reads

$$
r_{p_{T}}(\eta)=\frac{\operatorname{Cov}\left(\left[p_{T}\right]_{F},\left[p_{T}\right]_{-\eta}\right)}{\operatorname{Cov}\left(\left[p_{T}\right]_{F},\left[p_{T}\right]_{\eta}\right)} .
$$

For $\mathrm{Pb}+\mathrm{Pb}$ collisions we symmetrize the above expression in forward-backward directions to increase statistics. The forward bin is taken as $2.0<\eta<2.5$ and the center of the second bin of width 0.5 is moved from 0.1 to 1.6.

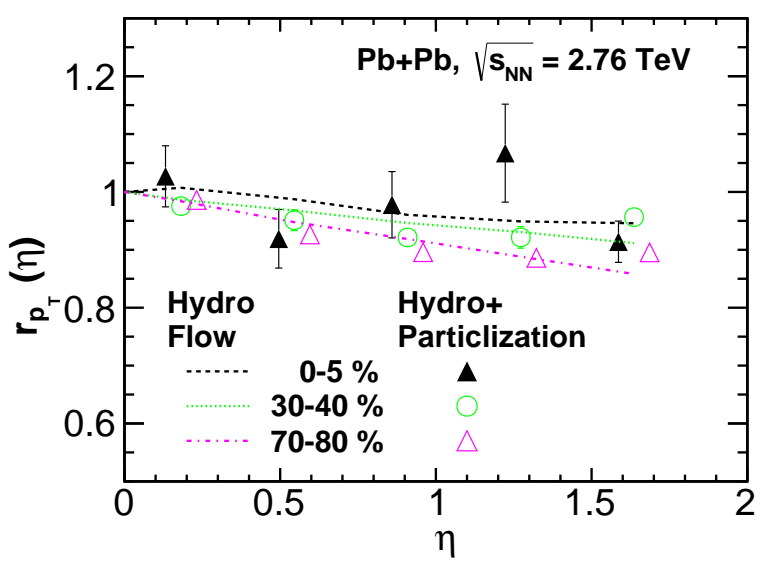

FIG. 7. (Color online) Factorization breaking coefficient $\mathrm{r}_{p_{T}}(\eta)$ of the average transverse momentum at different pseudorapidities. Symbols are for results from realistic finite multiplicity events and lines are obtained from spectra integration (see caption of Fig. 3).

In Fig. 7 is shown the result of the calculation of the 3-bin decorrelation measure $r_{p_{T}}(\eta)$ for three centralities (symbols). For centralities $30-40 \%$ and $70-80 \%$ the statistics is sufficient to indicate an increasing decorrelation as the bin separation increases. Similar results are obtained with oversampled events (lines), which gives a measure of the 3-bin decorrelation from collective flow only. We notice that the $p_{T}$-flow decorrelation in pseudorapidity increases when going from central to peripheral collisions.

\section{SUMMARY}

We investigate the event-by-event profile of the average transverse momentum of particles emitted in relativistic heavy-ion collisions. The model used consist of relativistic viscous hydrodynamics. The initial conditions for the evolution come from a Monte Carlo Glauber model with quark degrees of freedom. The model dynamics generates flow correlations from the collective expansion and non-flow correlations from resonance decays. We compare the hydrodynamic simulation results to preliminary ALICE Collaboration data on the Pearson correlation coefficient $b\left(\left[p_{T}\right]_{F},\left[p_{T}\right]_{B}\right)$. We find a fair agreement for the magnitude of the correlation, and qualitatively a similar centrality dependence.

We argue that the value Pearson correlation coefficient $b\left(\left[p_{T}\right]_{F},\left[p_{T}\right]_{B}\right)$ measured in $\mathrm{Pb}+\mathrm{Pb}$ collisions is dominated by statistical fluctuations of the average transverse momentum $\left[p_{T}\right]$ in finite multiplicity events. This problem can be reduced by using a definition of the variance of $\left[p_{T}\right]$ that excludes self-correlations in the definition of the correlation coefficient. The proposed transverse flowtransverse flow correlation coefficient gives an estimate of the correlation coefficient for the collective transverse 
flow in two pseudorapidity bins. The magnitude of this correlation is close to 1 in our calculation, several times larger than the standard Pearson correlation coefficient.

It would be interesting to measure in experiment the transverse flow-transverse flow correlation coefficient as a function of the pseudorapidity bin separation. It would be a measure of the correlations of the initial size of the fireball in the longitudinal direction. The results could also point to additional $p_{T}$ correlations in rapidity arising during the dynamic evolution of the fireball. We give an estimate of the 3-bin measure of $p_{T}$ decorrelation in pseudorapidity defined as the ratio of $p_{T}$ covariances cal- culated for different bin separations. With increasing bin separation $p_{T}-p_{T}$ correlation gets weaker. We find $5-10 \%$ decorrelation over two units of $\eta$.

\section{ACKNOWLEDGMENTS}

Research supported by the Polish Ministry of Science and Higher Education (MNiSW), by the National Science Centre grant 2015/17/B/ST2/00101, as well as by PLGrid Infrastructure.
[1] U. Heinz and R. Snellings, Ann.Rev.Nucl.Part.Sci. 63, 123 (2013), arXiv:1301.2826 [nucl-th]; C. Gale, S. Jeon, and B. Schenke, Int.J.Mod.Phys. A28, 1340011 (2013), arXiv:1301.5893 [nucl-th]; J.-Y. Ollitrault, J. Phys. Conf. Ser. 312, 012002 (2011), arXiv:1008.3323 [nucl-th].

[2] P. Bożek, W. Broniowski, and J. Moreira, Phys. Rev. C83, 034911 (2011), arXiv:1011.3354 [nucl-th]

[3] H. Petersen, V. Bhattacharya, S. A. Bass, and C. Greiner, Phys.Rev. C84, 054908 (2011), arXiv:1105.0340 [nucl-th],

[4] K. Xiao, F. Liu, and F. Wang, Phys.Rev. C87, 011901 (2013), arXiv:1208.1195 [nucl-th]

[5] J. Jia and P. Huo, Phys.Rev. C90, 034905 (2014) arXiv:1402.6680 [nucl-th].

[6] L.-G. Pang, G.-Y. Qin, V. Roy, X.-N. Wang, and G.L. Ma, Phys. Rev. C91, 044904 (2015), arXiv:1410.8690 [nucl-th]

[7] V. Khachatryan et al. (CMS Collaboration), Phys. Rev. C92, 034911 (2015), arXiv:1503.01692 [nucl-ex].

[8] G. Aad et al. (ATLAS Collaboration), (2017), ATLASCONF-2017-003

[9] B. B. Back et al. (PHOBOS), Phys. Rev. C74, 011901 (2006), arXiv:nucl-ex/0603026 [nucl-ex]; B. Abelev et al. (STAR Collaboration), Phys.Rev.Lett. 103, 172301 (2009), arXiv:0905.0237 [nucl-ex] G. Aad et al. (ATLAS Collaboration), (2015), ATLAS-CONF-2015-020

[10] M. Gazdzicki and S. Mrowczynski, Z. Phys. C54, 127 (1992).

[11] L. Stodolsky, Phys. Rev. Lett. 75, 1044 (1995).

[12] E. V. Shuryak, Phys. Lett. B423, 9 (1998), hep$\mathrm{ph} / 9704456$

[13] S. Mrowczynski, Phys. Lett. B430, 9 (1998), nuclth/9712030

[14] F. Liu, A. Tai, M. Gazdzicki, and R. Stock, Eur.Phys.J. C8, 649 (1999), arXiv:hep-ph/9809320 [hep-ph]

[15] S. A. Voloshin, V. Koch, and H. G. Ritter, Phys. Rev. C60, 024901 (1999), nucl-th/9903060

[16] G. Baym and H. Heiselberg, Phys. Lett. B469, 7 (1999), nucl-th/9905022.

[17] S. A. Voloshin (STAR Collaboration), AIP Conf. Proc. 610, 591 (2001) arXiv:nucl-ex/0109006.

[18] R. Korus, S. Mrowczynski, M. Rybczyński, and Z. Wlodarczyk, Phys. Rev. C64, 054908 (2001), nuclth/0106041

[19] J. Dias de Deus, E. Ferreiro, C. Pajares, and R. Ugoccioni, Eur.Phys.J. C40, 229 (2005), arXiv:hep- ph/0304068 [hep-ph]

[20] W. Broniowski, B. Hiller, W. Florkowski, and P. Bożek, Phys. Lett. B635, 290 (2006), arXiv:nucl-th/0510033 [nucl-th].

[21] M. Sharma and C. A. Pruneau, Phys. Rev. C79, 024905 (2009), arXiv:0810.0716 [nucl-ex]

[22] Y. Hama, R. P. G. Andrade, F. Grassi, W. L. Qian, and T. Kodama, Acta Phys. Polon. B40, 931 (2009), arXiv:0901.2849 [hep-ph].

[23] T. A. Trainor, Phys. Rev. C92, 024915 (2015) arXiv:1503.02197 [hep-ph]

[24] S. Basu, S. Chatterjee, R. Chatterjee, T. K. Nayak, and B. K. Nandi, Phys. Rev. C94, 044901 (2016), arXiv:1601.05631 [nucl-ex]

[25] S. Gavin, G. Moschelli, and C. Zin, (2016), arXiv:1612.07856 [nucl-th]

[26] Q. Liu and W.-Q. Zhao, (2016), arXiv:1611.02532 [hep$\mathrm{ph}]$.

[27] A. Bzdak, (2017), arXiv:1703.03003 [hep-ph]

[28] W. Broniowski, M. Chojnacki, and L. Obara, Phys. Rev. C80, 051902 (2009), arXiv:0907.3216 [nucl-th]

[29] P. Bożek and W. Broniowski, Phys. Rev. C85, 044910 (2012), arXiv:1203.1810 [nucl-th].

[30] A. Mazeliauskas and D. Teaney, Phys. Rev. C93, 024913 (2016), arXiv:1509.07492 [nucl-th]

[31] J. Adams et al. (STAR Collaboration), Phys. Rev. C71, 064906 (2005), arXiv:nucl-ex/0308033 [nucl-ex].

[32] D. Adamova et al. (CERES Collaboration), Nucl.Phys. A727, 97 (2003), arXiv:nucl-ex/0305002 [nucl-ex]

[33] S. Adler et al. (PHENIX Collaboration), Phys. Rev. Lett. 93, 092301 (2004), arXiv:nucl-ex/0310005 [nucl-ex]

[34] T. Anticic et al. (NA49 Collaboration), Phys. Rev. C70, 034902 (2004), arXiv:hep-ex/0311009 [hep-ex],

[35] B. B. Abelev et al. (ALICE), Eur. Phys. J. C74, 3077 (2014), arXiv:1407.5530 [nucl-ex]

[36] I. Altsybeev (ALICE Collaboration), poster presented at the Quark Matter conference, Chicago, Feb 5-11 (2017).

[37] V. Kovalenko and V. Vechernin, J. Phys. Conf. Ser. 798, 012053 (2017), arXiv:1611.07274 [nucl-th]

[38] B. Schenke, S. Jeon, and C. Gale, Phys. Rev. Lett. 106, 042301 (2011), arXiv:1009.3244 [hep-ph],

[39] P. Bożek, W. Broniowski, and M. Rybczyński, Phys. Rev. C94, 014902 (2016), arXiv:1604.07697 [nucl-th].

[40] T. Hirano and K. Tsuda, Phys. Rev. C66, 054905 (2002), arXiv:nucl-th/0205043.

[41] P. Bożek and W. Broniowski, Phys. Lett. B752, 206 
(2016), arXiv:1506.02817 [nucl-th]

[42] A. Monnai and B. Schenke, Phys. Lett. B752, 317 (2016), arXiv:1509.04103 [nucl-th],

[43] M. Chojnacki, A. Kisiel, W. Florkowski, and W. Broniowski, Comput. Phys. Commun. 183, 746 (2012), arXiv:1102.0273 [nucl-th].
[44] P. Bożek and W. Broniowski, (2017), arXiv:1701.09105 [nucl-th].

[45] P. Bożek, Phys. Rev. C93, 044908 (2016), arXiv:1601.04513 [nucl-th]

[46] F. G. Gardim, F. Grassi, M. Luzum, and J.-Y. Ollitrault, Phys.Rev. C87, 031901 (2013), arXiv:1211.0989 [nuclth] 